\title{
Modelagem computacional da resposta imune à vacina contra febre amarela
}

\author{
Carla R. B. Bonin ${ }^{1}$, Guilherme C. Fernandes ${ }^{2}$, Rodrigo W. dos $\operatorname{Santos}^{1}$, Marcelo Lobosco ${ }^{1}$ \\ ${ }^{1}$ Programa de Pós-graduação em Modelagem Computacional, \\ Universidade Federal de Juiz de Fora(UFJF), Juiz de Fora, Brasil \\ ${ }^{2}$ Faculdade de Medicina, Universidade Presidente Antônio Carlos, \\ Juiz de Fora, Brasil \\ rezendebonin@gmail.com
}

\begin{abstract}
An effective yellow fever vaccine has been available since 1937. Nevertheless, questions regarding its use remain poorly understood, such as the ideal dose to confer immunity against the disease, the need for booster dose, the optimal immunization schedule for immunocompetent, immunosuppressed, and children, among other issues. This work presents a computational model of the human immune response to vaccination against yellow fever that was able to replicate the levels of antibodies obtained experimentally in different vaccination scenarios, allowing a quantitative validation with experimental data. Some behaviors of the immune response described in the literature were also reproduced qualitatively.
\end{abstract}

Resumo. Desde 1937 está disponível uma vacina eficaz contra febre amarela. Ainda assim, questões relativas a seu uso permanecem pouco entendidas, como a dose ideal capaz de conferir imunidade contra a doença, a necessidade de dose reforço, o esquema ideal de vacinação para indivíduos imunocompetentes, imunossuprimidos, e crianças, dentre outras. Este trabalho apresenta um modelo computacional da resposta imune humana à vacinação contra a febre amarela que foi capaz de reproduzir os níveis de anticorpos obtidos experimentalmente em diferentes cenários relativos à vacinação, permitindo uma validação quantitativa com dados experimentais. Também foram reproduzidos qualitativamente alguns comportamentos da resposta imune descritos na literatura.

\section{Introdução}

Desde 1937 está disponível uma vacina eficaz contra a Febre Amarela (FA) (Theiler and Smith 1937). Apesar disso, ainda ocorrem grandes surtos em países da África e das Américas Central e do Sul. Nas últimas décadas, a FA tem sido registrada no Brasil além dos limites da área considerada endêmica (região amazônica). A partir de dezembro de 2017 foi registrado o maior surto de FA observado nas últimas décadas. Em virtude desse surto, percebeu-se a expansão da área de circulação do vírus amarílico em municípios que não eram considerados áreas de risco, principalmente nas proximidades das grandes capitais metropolitanas das regiões Sul e Sudeste do Brasil. Por esse motivo, 940 novos municípios foram acrescidos à lista de 3.529 municípios pertencentes à área com recomendação de vacinação (ACRV), totalizando 4.469 municípios. 
Pensando nos surtos recentes e na insuficiência dos estoques mundiais de vacina, a Organização Mundial da Saúde (OMS) lançou em abril de 2017 uma estratégia chamada Eliminate Yellow fever Epidemics (EYE), que visa eliminar as epidemias de FA no mundo até 2026 (WHO 2017). Para atingir os objetivos, a estratégia sugere ações em diferentes frentes, entre elas a pesquisa e o desenvolvimento para melhores ferramentas e práticas. É nesse cenário que a modelagem matemática e computacional se apresenta como uma possível ferramenta para auxiliar as pesquisas em vacinologia e em saúde pública.

O termo vacinologia computacional vem sendo empregado para descrever "tecnologias computacionais dedicadas a apoiar e melhorar o desenvolvimento de vacinas" (Söllner et al. 2010). Os modelos matemáticos têm sido utilizados há muitos anos para representar diversos aspectos do sistema imunológico e patologias relacionadas, porém suas aplicações para descrever os efeitos das vacinas têm sido bastante limitadas (Pappalardo et al. 2015) e quase sempre restritas às fases pré-clinicas dos estudos.

\section{Objetivo}

O objetivo deste trabalho é demonstrar que ferramentas matemático-computacionais podem ser utilizadas para simular a resposta imune de um indivíduo à vacina contra FA e através dessas ferramentas realizar experimentos associados a fase clínica com diferentes cenários e possibilidades em um ambiente totalmente computacional, sem a necessidade de grandes recursos financeiros ou em situações em que haja dificuldades metodológicas e/ou éticas para realização de estudos em humanos. Para isso, foi desenvolvido um modelo matemático-computacional da resposta imune à vacina contra FA. O modelo considera as principais populações de células e moléculas do Sistema Imune Humano (SIH) importantes no processo de aquisição de imunidade, como células apresentadoras de antígeno (APCs, do inglês Antigen Presenting Cells), linfócitos B e T, o vírus vacinal e os anticorpos, sendo que estes são considerados o principal marcador de imunidade.

\section{Contribuições do trabalho}

O modelo computacional proposto para representar a resposta imune à vacina contra FA foi capaz de reproduzir as concentrações de títulos de anticorpos encontrados experimentalmente em indivíduos imunocompetentes primovacinados com a dose padrão da vacina. Foram apresentados bons resultados para diversos outros cenários como indivíduos revacinados, portadores de doenças autoimunes sob uso de terapia imunomoduladora combinada e crianças. Além disso, foi possível reproduzir in silico os resultados experimentais da administração de diferentes doses da vacina contra FA observados na literatura (Martins et al. 2013). Foram simuladas também variações nas quantidades iniciais das células T, CD4+ e CD8+, contudo sem que fossem usados dados experimentais.

Após a simulação de todos esses cenários, muitos deles validados de forma quantitativa, pode-se concluir que o modelo desenvolvido (Bonin 2019) é uma ferramenta importante na investigação das variações de resposta imune entre diferentes indivíduos, podendo ser empregado na busca por melhores esquemas vacinais, na avaliação da necessidade de doses reforço e do melhor intervalo entre elas. Em suma, com um modelo que considera os principais personagens envolvidos na resposta imune humana à vacina contra FA é possível testar hipóteses e reduzir o espaço de busca por respostas às questões em aberto relativas à vacina. 


\section{Resultados e discussões}

Foram simulados diferentes cenários para comparar os resultados numéricos do modelo com os dados experimentais obtidos através da parceria com o Instituto René Rachou, Fiocruz Minas. Por questão de limitação de espaço, esta seção apresenta com maiores detalhes alguns dos resultados relacionados a dois dos cenários estudados: primeira vacinação e dose-reforço. Outros resultados para estes bem como para outros cenários podem ser encontrados na tese de doutorado da primeira autora deste artigo (Bonin 2019).

O primeiro cenário simulado foi o de um indivíduo adulto sendo vacinado pela primeira vez com a dose plena de um lote da vacina contra FA. Foi observado nos dados experimentais que, antes mesmo de os indivíduos serem vacinados, alguns deles já apresentam anticorpos contra FA. Existem algumas hipóteses para que isso aconteça, uma delas é a proteção cruzada causada pelo contato com outros flavivirus, como o vírus da dengue, por exemplo, ou uma vacinação anterior não registrada. Para que a simulação se aproximasse do cenário real, foi estabelecida como condição inicial do modelo uma concentração de anticorpos semelhante ao que foi obtido experimentalmente. Após a vacinação, foram medidos os níveis de anticorpos dos indivíduos que participaram do experimento em diferentes momentos. Essas amostras foram organizadas em grupos, de forma a ter indivíduos suficientes em cada grupo para que se pudesse observar o comportamento dos anticorpos ao longo do tempo. Esses grupos são: a) NV (dia 0), Naive, imediatamente antes da vacinação; b) PV (30-45 dias), de 30 a 45 dias após a vacinação; c) PV (1-5 anos), de 1 a 5 anos após a vacinação; d) PV (>5-9 anos), de mais de 5 até 9 anos após a vacinação; e e) PV (10 anos) - 10 anos ou mais após a vacinação.

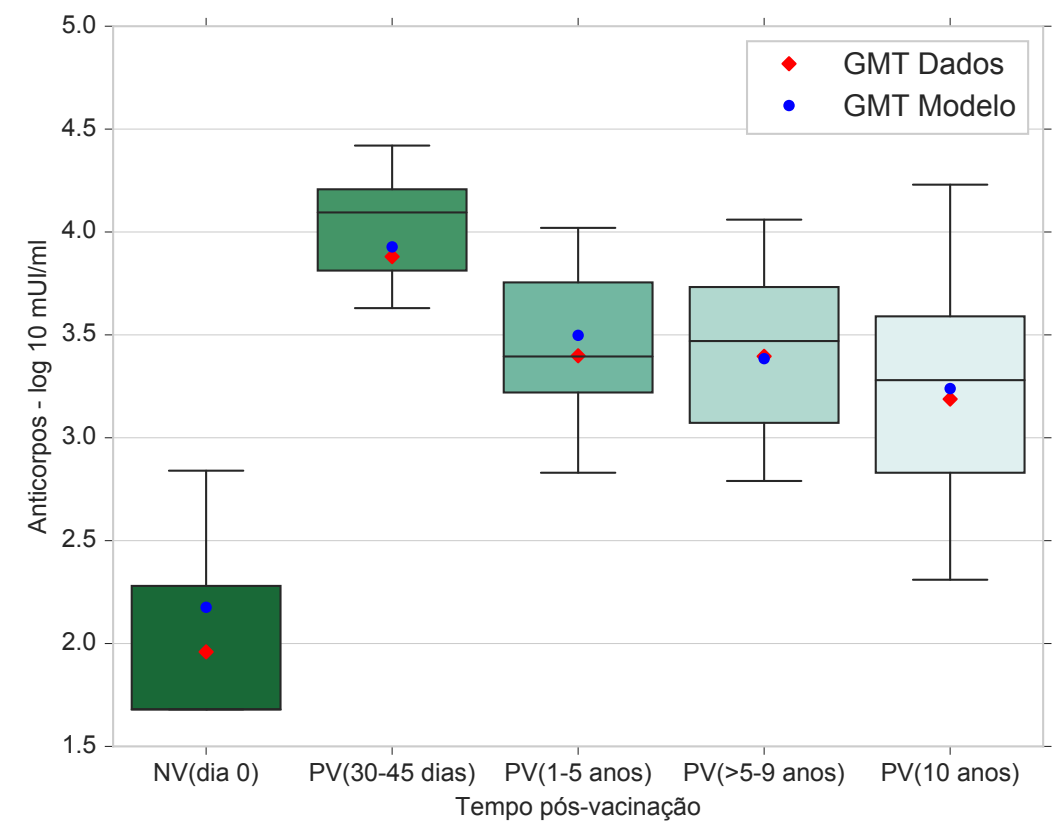

Figura 1. Intervalo interquartil (retângulos), mediana (linha preta), limite inferior e limite superior (hastes pretas) e médias geométricas (observadas e estimadas pelo modelo) de títulos de anticorpos para FA segundo tempo pós-vacinação. "GMT Dados" $(\diamond)$ refere-se à média geométrica dos dados experimentais e "GMT Modelo" (•) à média geométrica dos resultados computacionais. 
A Figura 1 apresenta a comparação entre os níveis de anticorpos obtidos numericamente e os dados experimentais, em diferentes intervalos de tempo pós-vacinação. Os erros numéricos para cada um destes intervalos foi 1,2\% (30-45 dias), 2,8\%(1-5 anos), 0,3\%(>5-9 anos) e 1,6\%(10 anos). Pode-se assim concluir que o modelo foi capaz de reproduzir quantitativamente os resultados experimentais.

O segundo cenário representa a revacinação de indivíduos adultos. Para verificar se o modelo é capaz de obter resultados semelhantes aos verificados experimentalmente após a aplicação da dose reforço, o seguinte método foi adotado. Inicialmente simulou-se um indivíduo primovacinado. Após simular o equivalente a 5.500 dias desde a aplicação da vacina, a simulação foi interrompida, salvaram-se as condições atuais de todas as populações do modelo e apenas a condição do vírus foi alterada, de zero para a dose padrão da vacina. Prosseguiu-se então a execução da aplicação para que esta simulasse mais 4.500 dias, ou seja, até esta atingir 10.000 dias. Os valores 5.500 e 10.000 dias foram escolhidos baseado nos dias em que existem dados experimentais disponíveis. $\mathrm{O}$ grupo PV (10 anos), ou seja, os indivíduos primovacinados 10 anos após a primeira dose, possui amostras coletadas até 5.475 dias (15 anos) após a vacinação. Por esse motivo, a dose reforço foi simulada no modelo 5.500 dias após a primeira dose. Da mesma forma, no grupo RV (10 anos), indivíduos 10 anos após a dose reforço, possui amostras coletadas 3.650 dias após a segunda dose, consequentemente 9.125 dias após a primeira dose. Por esse motivo, foram simulados 10.000 dias após a aplicação da primeira dose.

A Figura 2 apresenta o resultado da simulação. Neste cenário, os resultados numéricos não estão na mesma unidade dos experimentais. Por esse motivo, os gráficos que apresentam esses resultados possuem dois eixos Y, cada um representando uma unidade: um para os resultados numéricos e outro para os experimentais. Dessa forma, é possível comparar qualitativamente o comportamento experimental com o obtido numericamente. Pode-se observar que o modelo consegue reproduzir qualitativamente o aumento no nível de anticorpos observado após a administração da dose reforço, bem como seu posterior decaimento.

Existem situações em que a resposta imune de alguns indivíduos difere da resposta habitualmente obtida pela vacinação em adultos imunocompetentes. Foram obtidos dados experimentais para duas dessas situações. O primeiro caso é o da vacinação de crianças e o segundo é o da vacinação de indivíduos com doenças autoimunes sob uso de terapia imunomoduladora. Foram feitas alterações nos valores de alguns parâmetros do modelo na tentativa de reproduzir a resposta imune nesses dois casos. Os parâmetros que tiveram seus valores alterados foram escolhidos baseado em informações descritas na literatura. Em ambas as situações, os resultados numéricos conseguiram reproduzir o comportamento observado experimentalmente (Bonin 2019).

Baseado em estudos nos quais foram avaliadas as respostas imunes de voluntários à doses da vacina contra FA menores que a padrão usada atualmente (Martins et al. 2013; Martins et al. 2018), foi feita outra simulação. Nela, avaliam-se os resultados numéricos quando estas mesmas diferentes doses são simuladas. Os erros ficaram abaixo de 2,5\% para 5 das 6 doses testadas, o que demonstra que o modelo, ajustado usando apenas os dados de indivíduos primovacinados com a dose padrão, foi capaz de reproduzir de forma satisfatória a resposta imune obtida com a vacinação usando doses menores que a padrão. 


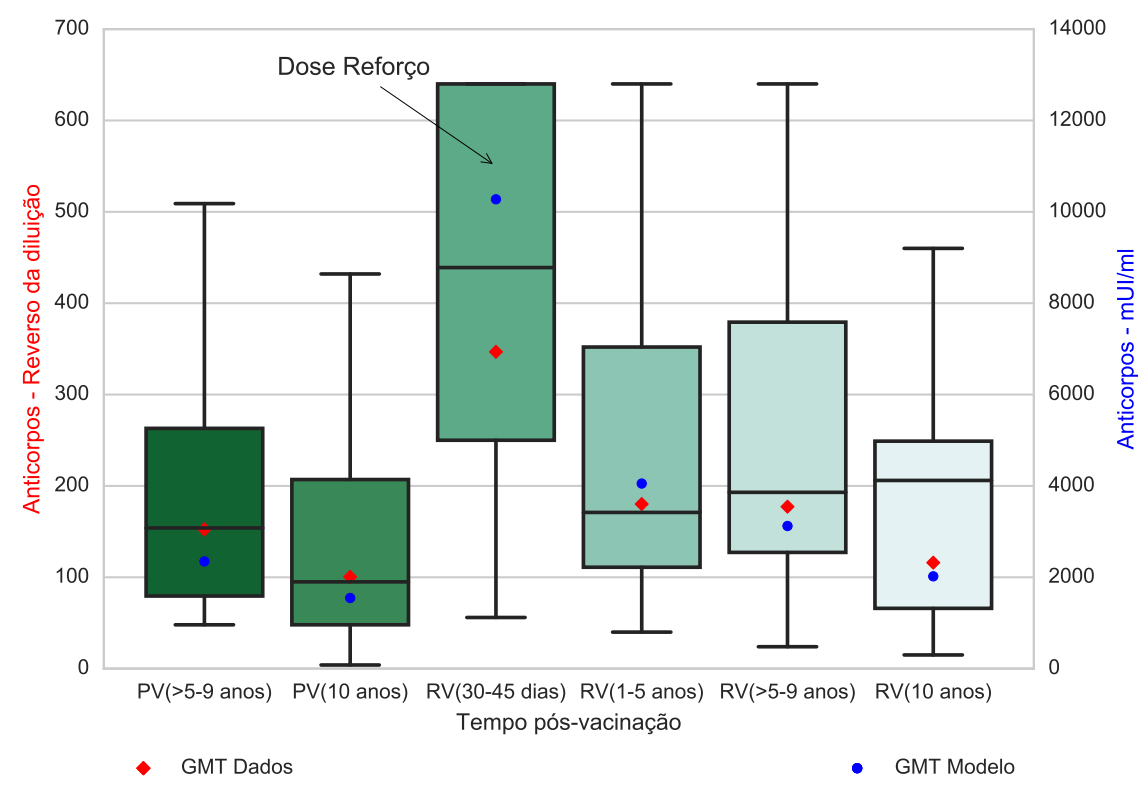

Figura 2. Intervalo interquartil (retângulos), mediana (linha preta), limite inferior e limite superior (hastes pretas) e médias geométricas (observadas e estimadas pelo modelo) de títulos de anticorpos para FA segundo tempo pós-vacinação, primeira dose e dose reforço. "GMT Dados" ( $\diamond)$ refere-se à média geométrica dos dados e "GMT Modelo" (•) à média geométrica dos resultados computacionais.

Todos os cenários descritos anteriormente serviram para validar o modelo. A fim de testar hipóteses e avaliar comportamentos descritos na literatura, foram realizadas simulações da variação na quantidade de células T CD4 e T CD8 no organismo. Deseja-se avaliar o impacto dessas populações de células nas curvas de interesse, viremia e anticorpos. Para esses casos não foram utilizados dados experimentais e por esse motivo apenas o comportamento qualitativo foi avaliado e comparado ao que é descrito na literatura. Para o primeiro caso, a simulação de uma redução de $90 \%$ na contagem inicial de células T CD4+ mostrou que os níveis de anticorpos gerados ficam abaixo do nível mínimo considerado protetor, antes mesmo de completar um ano após a vacinação. Esse resultado pode reforçar a contraindicação de vacinação para indivíduos com contagem de células T CD4+ abaixo de $2 \times 10^{5}$ células $/ \mathrm{ml}$. Para o cenário da variação na quantidade inicial de células T CD8+, as curvas que apresentam as reduções nestas populações de $25 \%$ e $50 \%$ mostraram um aumento significativo da viremia, que ultrapassou o nível detectável de aproximadamente 2.147 cópias $/ \mathrm{ml}$. Esse resultado está de acordo com o que foi descrito na literatura (Reinhardt et al. 1998).

\section{Conclusões}

Este trabalho apresentou a validação quantitativa e qualitativa do modelo matemáticocomputacional para representar a resposta imune à vacina contra FA usando cinco cenários distintos: primo-vacinação em adultos, dose-resposta, dose-reforço em adultos, vacinação em indivíduos em uso de terapia imunomoduladora e primo-vacinação em crianças. Os resultados numéricos foram coletados e comparados aos experimentais, sendo os maiores erros observados iguais a 12,9\%. Para outros resultados que não puderam ser comparados diretamente, por serem utilizadas unidades distintas, observou-se 
que os resultados numéricos obtidos pelo modelo computacional reproduzem satisfatoriamente o comportamento observado nos dados experimentais.

Os experimentos numéricos mostram que, entre todas as doses de vacina avaliadas, a mais baixa capaz de conferir imunidade contra a FA é cerca de metade da dose de referência, 587 UI. Os resultados também sugerem que a hipótese de que a imaturidade imunológica em crianças limita a persistência de plasmócitos de vida longa não está relacionada ao decaimento de anticorpos observado experimentalmente. Os experimentos numéricos mostram que esse fenômeno se deve à menor indução de plasmócitos de vida longa. Finalmente, o decaimento observado no nível de anticorpos no período de 10 anos após a vacinação sugere que é necessária uma dose de reforço para manter a imunidade contra a FA.

Embora o modelo apresentado neste trabalho se concentre na vacina contra a febre amarela, acreditamos que ele possa ser usado para reproduzir a resposta do sistema imune a outras doenças. Modelos como o apresentado neste trabalho podem, em um futuro próximo, ser usados para ajudar a responder a perguntas que, esperamos, serão feitas em um futuro próximo: qual é a dose mínima da vacina COVID-19 para proteger contra a doença?

\section{Referências}

[Bonin 2019] Bonin, C. R. B. (2019). Modelagem Computacional da Resposta Imune à Vacina Contra a Febre Amarela. PhD thesis, Programa de Pós-Graduação em Modelagem Computacional, Universidade Federal de Juiz de Fora.

[Martins et al. 2018] Martins, R. d. M., Maia, M. d. L. S., Lima, S. M. B. d., Noronha, T. G. d., Xavier, J. R., Camacho, L. A. B., Albuquerque, E. M. d., Farias, R. H. G., Castro, T. d. M. d., Homma, A., et al. (2018). Duration of post-vaccination immunity to yellow fever in volunteers eight years after a dose-response study. Vaccine, 36(28):4112-4117.

[Martins et al. 2013] Martins, R. M., Maia, M. d. L. S., Farias, R. H. G., Camacho, L. A. B., Freire, M. S., Galler, R., Yamamura, A. M. Y., Almeida, L. F. C., Lima, S. M. B., Nogueira, R. M. R., et al. (2013). 17dd yellow fever vaccine: a double blind, randomized clinical trial of immunogenicity and safety on a dose-response study. $\mathrm{Hu}$ man vaccines \& immunotherapeutics, 9(4):879-888.

[Pappalardo et al. 2015] Pappalardo, F., Flower, D., Russo, G., Pennisi, M., and Motta, S. (2015). Computational modelling approaches to vaccinology. Pharmacological research, 92:40-45.

[Reinhardt et al. 1998] Reinhardt, B., Jaspert, R., Niedrig, M., Kostner, C., and L'ageStehr, J. (1998). Development of viremia and humoral and cellular parameters of immune activation after vaccination with yellow fever virus strain $17 \mathrm{~d}$ : a model of human flavivirus infection. Journal of medical virology, 56(2):159-167.

[Söllner et al. 2010] Söllner, J., Heinzel, A., Summer, G., Fechete, R., Stipkovits, L., Szathmary, S., and Mayer, B. (2010). Concept and application of a computational vaccinology workflow. Immunome research, 6(2):S7.

[Theiler and Smith 1937] Theiler, M. and Smith, H. H. (1937). The use of yellow fever virus modified by in vitro cultivation for human immunization. J. Exp. Med., 65(6):787-800.

[WHO 2017] WHO (2017). Weekly epidemiological record. 